\title{
Simultaneous determination of polyphenol content Vitis amurensis Rupr. by tandem mass spectrometry
}

\author{
Mayya P. Razgonova ${ }^{1,2, *}$, Evgeniy I. Kislin ${ }^{1}$, Andrey Sh. Sabitov ${ }^{1}$, Elena V. Perminova ${ }^{1}$, \\ Natalia M. Mikhailova ${ }^{1}$, Kirill S. Golokhvast ${ }^{1,3,4}$ \\ ${ }^{1}$ N.I. Vavilov All-Russian Institute of Plant Genetic Resources, B. Morskaya 42-44, 190000, Saint- \\ Petersburg, Russian Federation \\ ${ }^{2}$ Far Eastern Federal University, Sukhanova 8, 690950, Vladivostok, Russian Federation \\ ${ }^{3}$ Pacific Geographical Institute, Far Eastern Branch of the Russian Academy of Sciences, Radio 7, \\ 690041, Vladivostok, Russian Federation \\ ${ }^{4}$ Siberian Federal Scientific Centre of Agrobiotechnology, 633501, Centralnaya, Presidium, \\ Krasnoobsk
}

\begin{abstract}
Vitis amurensis Ruprecht contains a large number of polyphenolic compounds which are biologically active components. For the most efficient and safe extraction supercritical carbon dioxide was used. In this work, for the first time, a comparative metabolomic study of biologically active substances of wild grapes collected from five different places of the Primorsky and Khabarovsk territories is carried out. To identify target analytes in ethanol extracts of grape berries, high performance liquid chromatography (HPLC) was used in combination with an amaZon SL ion trap (manufactured by BRUKER DALTONIKS, Germany) equipped with an ESI electrospray ionization source in negative and positive ion modes. The mass spectrometer was used in the scan range $m / z 100-1.700$ for MS and MS / MS. Used fragmentation of the $4^{\text {th }}$ order. Primary mass spectrometric results showed the presence of 94 biologically active compounds corresponding to the species $V$. amurensis, moreover, salvianolic acids F, D and G, oleanoic, ursolic, myristoleic acids, berbericinin, mearnsetin, esculin, nevadensin, stigmasterol, fucosterol, phlorizin, L-tryptophan identified for the first time in $V$. amurensis.
\end{abstract}

\section{Introduction}

The appearance of the first representatives of the Vitaceae family, belonging to the genus Vitis, must undoubtedly be attributed to the Upper Cretaceous period, when there were already types of plants very similar in leaves to vines. The absence of seeds does not allow, however, in many cases to have complete confidence in their belonging to the genus Vitis. $[1,51]$.

These types include the Vitis dakotana Berry vine found in the Upper Cretaceous deposits found in Harding County in South Dakota, which is very similar in appearance to modern

\footnotetext{
* Corresponding author: m.razgonova@vir.nw.ru
} 
vines. [2, 3]. The evolution of grape plants approaching the cultivated vine, judging by the fossil finds, took place especially intensively in Central and Southern Europe during the second half of the Tertiary period and then especially in the Quaternary period. On the territory of Russia, quite a few finds of fossils are also known belonging to the genera Cissites, Ampelopsis, Parthenocissus, and especially to the genus Vitis: V. sachalinensis Krysht. and V. crenata Heer on Sakhalin, V. teutonica A. Br. - near Taganrog and on the Irtysh River, as well as $V$. praevinifera Sap. - on the Krynka river. All these data show that the evolution of the vine on the territory of Russia proceeded from ancient times. This can also be seen from the fact that now the wild grape $V$. sylvestris Gmel grows in many regions of Russia [21, 22, 23].

There is very little information about the culture of East Asian grape varieties. $V$. lanata Roxb is cultivated in eastern India. and $V$. tomentosa Heyne, in Japan and Korea $-V$. Thunbergii Sieb. et Zucc. called V. Seiboldii hort [15].

More complete information is available regarding $V$. amurensis Rupr., which was first introduced into the culture by I.V. Michurin. In his work "Results of half a century of work" I.V. Michurin describes four forms of $V$. amurensis Rupr., which were isolated in the Far East [29, 30].

Ripe berries, fruit skins, ridges, leaves, seeds, vine bark, red grape wine are used to isolate biologically active substances. Fruits contain $65-85 \%$ water, $10-33 \%$ sugar (glucose and fructose), phlobaphene, gallic acid, quercetin, enin, glycosides - monodelphinidin and didelphinidin, acids (malic, silicic, salicylic, phosphoric, tartaric, citric, etc.) pectin and tannins, potassium, magnesium, calcium, manganese, cobalt, iron and vitamins: $\mathrm{B}_{1}, \mathrm{~B}_{2}, \mathrm{~B}_{6}$, $\mathrm{B}_{12}, \mathrm{~A}, \mathrm{C}, \mathrm{P}, \mathrm{PP}$, folic acid, and enzymes.

The dominant class of biologically active compounds of grape, and especially grape ridges, are bioflavonoids and, in particular, the so-called complexes of oligomeric proanthocyanidins or condensed tannins, which are polymeric forms of flavonoids from the class of catechins [44].

In European medicine, until recently, grapes were widely used as a means of therapy and rehabilitation for a wide range of diseases: chronic recurrent inflammatory processes, tuberculosis, kidney disease, arterial hypertension, etc. The aim of this work was a comparative metabolomic study of biologically active substances of wild grapes collected in five different places in the Far Eastern taiga in the Primorsky and Khabarovsk territories (Fig. 1). High-performance liquid chromatography in combination with a BRUKER DALTONIKS ion trap (tandem mass spectrometry) was used to identify target analytes in extracts. This work presents a detailed study of the metabolomic composition of grape juice taken from five habitats of $V$. amurensis in the Far East: Pakhtusov Islands and Rikorda Island (Peter the Great Bay, Japanese Sea), the vicinity of Artem (Primorsky Territory), the vicinity of the river Arsenyevka (Primorsky Territory), environs of Vyazemsky (Khabarovsk Territory).

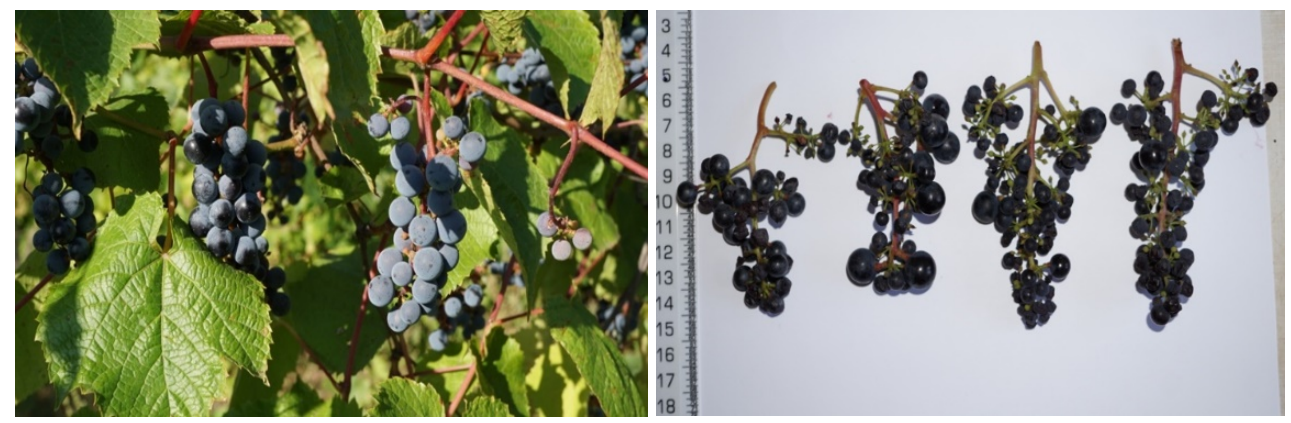

Fig.1. Wild grape $V$. amurensis harvested in the vicinity of Artem (Primorsky Territory). 


\section{Materials and Methods}

\subsection{Materials}

The object of the study was the berries of the wild grape V. amurensis, collected in the floodplain of the Arsenyevka River, Primorsky Territory (N. 44 ${ }^{\circ} 52^{\prime} 18^{\prime \prime}$, E $\left.133^{\circ} 35^{\prime} 12^{\prime \prime}\right)$ in the vicinity of Vyazemsky, Khabarovsk Territory (N 47 $32^{\prime} 15^{\prime \prime}$, E $\left.134^{\circ} 45^{\prime} 20^{\prime \prime}\right)$, in the vicinity of Artem, Primorsky Territory (N 4321'34 ", E 132 $\left.11^{\prime} 19^{\prime \prime}\right)$, on Rikord Island, Peter the Great Bay, Sea of Japan (N 42 $52^{\prime} 54^{\prime \prime}$, E $131^{\circ} 40^{\prime} 06^{\prime \prime}$ ), on the Pakhtusov Islands, Peter the Great Bay, Sea of Japan (N 42 53'57”, E 131 $38^{\prime} 45^{\prime \prime}$ ). The grapes were harvested at the end of August and September 2020. All samples morphologically corresponded to the pharmacopoeial standards of the State Pharmacopoeia of the Russian Federation [45].

\subsection{Methods}

\subsubsection{Fractional maceration}

To obtain highly concentrated extracts, fractional maceration was applied. In this case, the total amount of the extractant (ethyl alcohol of reagent grade) is divided into 3 parts and is consistently infused on grapes with the first part, then with the second and third. The infusion time of each part of the extractant was 7 days.

\subsubsection{Liquid chromatography}

HPLC was performed using Shimadzu LC-20 Prominence HPLC (Shimadzu, Japan) was used, equipped with an UV-sensor and a Shodex ODP-40 4E reverse phase column to perform the separation of multicomponent mixtures. The gradient elution program was as follows: 0.01-4 min, $100 \% \mathrm{CH}_{3} \mathrm{CN}$; 4-60 min, $100-25 \% \mathrm{CH}_{3} \mathrm{CN}$; $60-75 \mathrm{~min}, 25-0 \% \mathrm{CH}_{3} \mathrm{CN}$; control washing 75-120 min $0 \% \mathrm{CH}_{3} \mathrm{CN}$. The entire HPLC analysis was done with a UVdetector at wavelengths of $230 \eta \mathrm{m}$ and $330 \mathrm{\eta m}$; the temperature corresponded to $17^{\circ} \mathrm{C}$. The injection volume was $1 \mathrm{ml}$.

\subsubsection{Tandem mass spectrometry}

MS analysis was performed on an ion trap amaZon SL (BRUKER DALTONIKS, Germany) equipped with an ESI source in negative ion mode. The optimized parameters were obtained as follows: ionization source temperature: $70^{\circ} \mathrm{C}$, gas flow: $41 / \mathrm{min}$, nebulizer gas (atomizer): 7.3 psi, capillary voltage: $4500 \mathrm{~V}$, end plate bend voltage: $1500 \mathrm{~V}$, fragmentary: $280 \mathrm{~V}$, collision energy: $60 \mathrm{eV}$. An ion trap was used in the scan range m / z $100-1.700$ for $\mathrm{MS}$ and MS/MS. The capture rate was one spectrum/s for MS and two spectrum/s for MS/MS. Data collection was controlled by Windows software for BRUKER DALTONIKS. All experiments were repeated three times. A two-stage ion separation mode (MS/MS mode) was implemented.

\section{Results and discussion}

Ten of the most consumed extracts of $V$. amurensis have been selected. All of them have a rich polyphenolic composition. There were six extracts from wild $V$. amurensis: Arsenyevka River (Primorsky Territory), Vyazemsky (Khabarovsk Territory), Artem (Primorsky Territory), Rikord Island and Pakhtusov Islands, Peter the Great Bay, Japanese Sea. High 
accuracy mass spectrometric data were recorded on an ion trap amaZon SL BRUKER DALTONIKS equipped with an ESI source in the mode of negative ions. The four-stage ion separation mode (MS/MS mode) was implemented. All the chemical profiles of the samples were obtained by HPLC - ESI - MS/MS method. A total of 300 peaks were detected in the chromatogram (Fig.2). The combination of both ionization modes (positive and negative) in MS full scan mode gave extra certainly to the molecular mass determination. The negative ion mode provides the highest sensitivity and results in limited fragmentation, making it most suited to infer the molecular mass of the separated polyphenols, especially in cases where concentration is low. By comparing the $\mathrm{m} / \mathrm{z}$ values, the RT and the fragmentation patterns with the $\mathrm{MS}^{2}$ spectral data taken from the literature $[7 ; 11 ; 12 ; 13 ; 24 ; 35 ; 46 ; 47 ; 49 ; 53$; etc] or to search the data bases (MS2T, MassBank, HMDB). A unifying system table was compiled of the molecular masses of the target analytes isolated from the EtOH-extract of $V$. amurensis for ease of identification (Table 1). The 94 polyphenols shown in Table 1 belong to different polyphenolic families: anthocyanidins, flavones, flavonols, flavan-3-ols, flavanones, hydroxycinnamic acids, hydroxybenzoic acids, stilbenes, tannins.

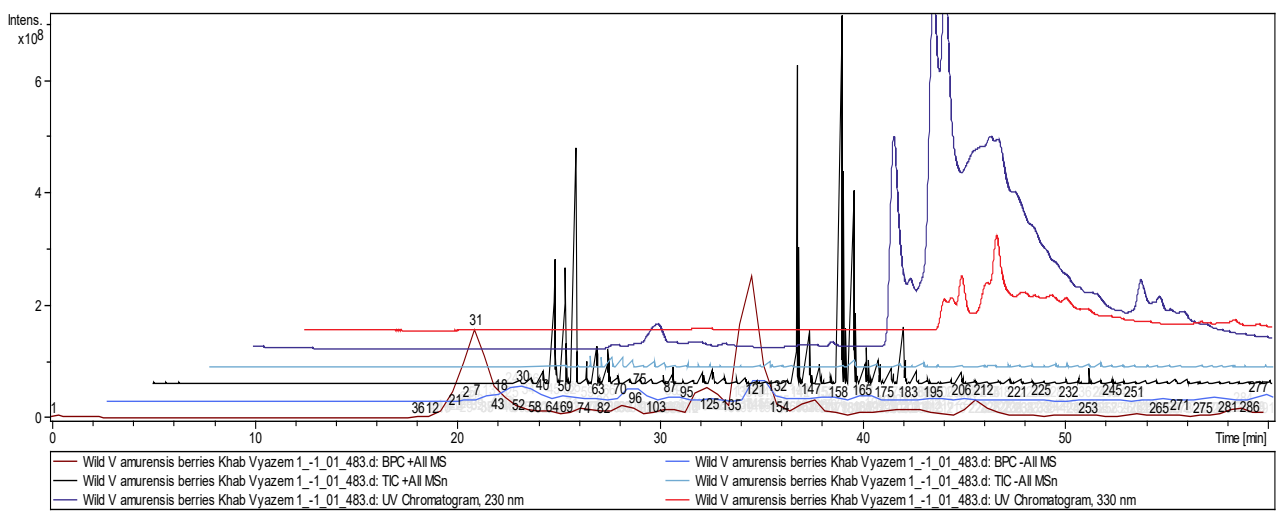

Fig.2. Chemical profiles of the V. amurensis (Vyazemsky, Khabarovsk Territory) sample represented total ion chromatogram from EtOH-extract.

A standardized system table of molecular weights and fragmented ions of target analytes isolated in extracts of Vitis amurensis Rupr. was compiled from the results of measurements (Table 1).

Table 1. Metabolomic analysis of biologically active substances from extracts of Vitis amurensis Rupr.

\begin{tabular}{|c|c|c|c|c|c|c|c|c|}
\hline № & $\begin{array}{c}\text { Class of } \\
\text { compounds }\end{array}$ & Identification & Formula & $\begin{array}{l}\text { Molecula } \\
\text { r mass }\end{array}$ & $\begin{array}{c}\text { Observe } \\
\text { d mass } \\
{[\mathrm{M}-\mathrm{H}]-}\end{array}$ & $\begin{array}{c}\text { Observe } \\
\text { d mass } \\
{[\mathbf{M}+\mathbf{H}]+}\end{array}$ & $\begin{array}{c}\text { MS/MS } \\
\text { fragmenta } \\
\text { tion }\end{array}$ & References \\
\hline 1 & $\begin{array}{c}\text { Anthocyani } \\
\mathbf{n}\end{array}$ & $\begin{array}{c}\text { Pelargonidin-3-O- } \\
\text { glucoside (callistephin) }\end{array}$ & $\mathrm{C}_{21} \mathrm{H}_{21} \mathrm{O}_{10}$ & 433.3854 & & 433 & $\begin{array}{l}414 ; 271 \\
172 ; 116\end{array}$ & {$[7 ; 47 ; 53]$} \\
\hline 2 & $\begin{array}{c}\text { Anthocyani } \\
\text { n }\end{array}$ & Peonidin-3-O-glucoside & $\mathrm{C}_{22} \mathrm{H}_{23} \mathrm{O}_{11+}$ & 463.4114 & & 463 & $\begin{array}{l}301 ; 286 \\
258 ; 230 \\
202 ; 174 \\
\end{array}$ & {$[12 ; 24 ; 53]$} \\
\hline
\end{tabular}




\begin{tabular}{|c|c|c|c|c|c|c|c|c|}
\hline 5 & $\begin{array}{c}\text { Anthocyani } \\
\mathrm{n}\end{array}$ & $\begin{array}{c}\text { Cyanidin 3,5-O- } \\
\text { dihexoside }\end{array}$ & $\mathrm{C}_{27} \mathrm{H}_{31} \mathrm{O}_{16}$ & 611.5335 & & 611 & $287 ; 449$ & {$[7 ; 35]$} \\
\hline 6 & $\begin{array}{c}\text { Anthocyani } \\
n\end{array}$ & $\begin{array}{l}\text { Cyanidin 3,5-O- } \\
\text { diglucoside }\end{array}$ & $\mathrm{C}_{27} \mathrm{H}_{31} \mathrm{O}_{16}$ & 611.5335 & & 611 & $287 ; 449$ & {$[24 ; 53]$} \\
\hline 7 & $\begin{array}{c}\text { Anthocyani } \\
\mathbf{n}\end{array}$ & $\begin{array}{l}\text { Peonidin-3,5-diglucoside } \\
\text { [Peonin; Peonidin 3- } \\
\text { Glucoside-5-Glucoside] }\end{array}$ & $\mathrm{C}_{28} \mathrm{H}_{33} \mathrm{O}_{16}$ & 625.5520 & & 625 & $\begin{array}{l}301 ; 463 \\
286 ; 258\end{array}$ & {$[24 ; 53]$} \\
\hline 8 & $\begin{array}{c}\text { Anthocyani } \\
\mathbf{n}\end{array}$ & $\begin{array}{c}\text { Malvidin 3-(6-O- } \\
\text { coumaroyl)glucoside }\end{array}$ & $\mathrm{C}_{32} \mathrm{H}_{31} \mathrm{O}_{14}$ & 639.5801 & & 639 & $\begin{array}{l}331 ; 315 \\
299 ; 270 \\
242 ; 179\end{array}$ & {$[12 ; 24 ; 53]$} \\
\hline 9 & $\begin{array}{c}\text { Anthocyani } \\
\mathbf{n}\end{array}$ & $\begin{array}{c}\text { Petunidin 3-O- } \\
\text { glucoside-5- } \boldsymbol{O} \text {-glucoside } \\
\text { [Petunidin } 3,5 \text {-di- } O \text {-beta- } \\
\text { D-glucoside] }\end{array}$ & $\mathrm{C}_{28} \mathrm{H}_{33} \mathrm{O}_{17}$ & 641.5514 & & 641 & $\begin{array}{l}317 ; 479 \\
420 ; 257 \\
302 ; 274\end{array}$ & {$[24 ; 53]$} \\
\hline 10 & $\begin{array}{c}\text { Anthocyani } \\
\text { n }\end{array}$ & $\begin{array}{l}\text { Malvidin 3-(6'-p- } \\
\text { caffeoylglucoside) }\end{array}$ & $\mathrm{C}_{32} \mathrm{H}_{31} \mathrm{O}_{15}$ & 655.5795 & & 655 & $\begin{array}{c}493 ; 331 \\
315 ; 313 \\
179\end{array}$ & {$[11 ; 12]$} \\
\hline 11 & $\underset{\mathbf{n}}{\text { Anthocyani }}$ & Malvidin 3,5-diglucoside & $\mathrm{C}_{32} \mathrm{H}_{31} \mathrm{O}_{15}$ & 655.5795 & & 655 & $\begin{array}{l}331 ; 493 \\
299 ; 179\end{array}$ & {$[24 ; 53]$} \\
\hline 12 & $\begin{array}{c}\text { Anthocyani } \\
\mathbf{n}\end{array}$ & $\begin{array}{l}\text { Malvidin 3-acetyl-5- } \\
\text { glucoside }\end{array}$ & $\mathrm{C}_{31} \mathrm{H}_{37} \mathrm{O}_{18+}$ & 697.6147 & & 697 & $\begin{array}{c}535 ; 493 \\
331 ; 315 \\
299 ; 242 \\
179\end{array}$ & [53] \\
\hline 13 & $\begin{array}{c}\text { Anthocyani } \\
\mathbf{n}\end{array}$ & $\begin{array}{l}\text { Petunidin 3- } \\
\text { coumaroylglucoside-5- } \\
O \text {-glucoside }\end{array}$ & $\mathrm{C}_{34} \mathrm{H}_{43} \mathrm{O}_{21}$ & 787.6926 & & 787 & $\begin{array}{c}625 ; 479 \\
317 ; 302 \\
301 ; 274 \\
\quad 246\end{array}$ & {$[24 ; 53]$} \\
\hline 14 & $\begin{array}{c}\text { Anthocyani } \\
\mathbf{n}\end{array}$ & $\begin{array}{c}\text { Malvidin 3- } \\
\text { coumaroylglucoside-5- } \\
O \text {-glucoside }\end{array}$ & $\mathrm{C}_{35} \mathrm{H}_{45} \mathrm{O}_{21}$ & 801.7192 & & 801 & $\begin{array}{l}639 ; 493 \\
331 ; 315 \\
287 ; 270\end{array}$ & [53] \\
\hline 15 & Tannin & $\begin{array}{l}\text { Procyanidin A-type } \\
\text { dimer }\end{array}$ & $\mathrm{C}_{30} \mathrm{H}_{24} \mathrm{O}_{12}$ & 576.501 & & 577 & $\begin{array}{l}425 ; 397 ; \\
373 ; 287 ; \\
245 ; 181\end{array}$ & {$[12 ; 37 ; 49]$} \\
\hline 16 & Flavone & Apigenin & $\mathrm{C}_{15} \mathrm{H}_{10} \mathrm{O}_{5}$ & 270.2369 & & 271 & $\begin{array}{c}253 ; 181 \\
137\end{array}$ & {$[46 ; 54]$} \\
\hline 17 & Flavone & Luteolin & $\mathrm{C}_{15} \mathrm{H}_{10} \mathrm{O}_{6}$ & 286.2363 & & 287 & $\begin{array}{l}271 ; 225 \\
175 ; 158\end{array}$ & {$[18 ; 28 ; 48]$} \\
\hline 18 & Flavone & Nevadensin & $\mathrm{C}_{18} \mathrm{H}_{16} \mathrm{O}_{7}$ & 344.3154 & 343 & & $\begin{array}{l}328 ; 313 \\
269 ; 259\end{array}$ & {$[32 ; 56]$} \\
\hline 19 & Flavone & Apigenin-7-O-glucoside & $\mathrm{C}_{21} \mathrm{H}_{20} \mathrm{O}_{10}$ & 432.3775 & & 433 & $\begin{array}{c}414 ; 287 \\
241 ; 186 \\
158\end{array}$ & $\begin{array}{l}{[12 ; 18 ; 27 ;} \\
46]\end{array}$ \\
\hline 20 & Flavone & $\begin{array}{c}\text { Isovitexin 6"-O- } \\
\text { deoxyhexoside [Apigenin } \\
\text { 6-C-glucoside 6"-O- } \\
\text { deoxyhexoside] }\end{array}$ & $\mathrm{C}_{27} \mathrm{H}_{30} \mathrm{O}_{14}$ & 578.5187 & & 579 & $\begin{array}{c}415 ; 397 \\
344 ; 297 \\
177\end{array}$ & {$[31]$} \\
\hline 21 & Flavone & $\begin{array}{c}\text { Vitexin 2"-O-glucoside } \\
\text { [Apigenin 8-C-glucoside } \\
\text { 2"-O-glucoside] }\end{array}$ & $\mathrm{C}_{27} \mathrm{H}_{30} \mathrm{O}_{15}$ & 594.5181 & & 595 & $\begin{array}{c}415 ; 353 \\
283 ; 265 \\
176\end{array}$ & [31] \\
\hline 22 & Flavone & $\begin{array}{c}\text { Apigenin 6- } C \text {-[6"-acetyl- } \\
\text { 2"-O-deoxyhexoside]- } \\
\text { glucoside }\end{array}$ & $\mathrm{C}_{29} \mathrm{H}_{32} \mathrm{O}_{15}$ & 620.5554 & & 621 & $\begin{array}{c}561 ; 547 \\
533 ; 461 \\
\quad 433 \\
\end{array}$ & [31] \\
\hline 23 & Flavonol & Kaempferol & $\mathrm{C}_{15} \mathrm{H}_{10} \mathrm{O}_{6}$ & 286.2363 & & 287 & $\begin{array}{l}269 ; 227 \\
\quad 153\end{array}$ & $\begin{array}{c}{[12 ; 27 ; 32 ;} \\
55]\end{array}$ \\
\hline 24 & Flavonol & Dihydrokaempferol & $\mathrm{C}_{15} \mathrm{H}_{12} \mathrm{O}_{6}$ & 288.2522 & & 289 & $\begin{array}{c}271 ; 243 \\
199 ; 189 \\
127\end{array}$ & {$[8 ; 26 ; 40]$} \\
\hline 25 & Flavonol & Kaempferide & $\mathrm{C}_{16} \mathrm{H}_{12} \mathrm{O}_{6}$ & 300.2629 & & 301 & $\begin{array}{c}283 ; 265 \\
239 ; 211 \\
185\end{array}$ & {$[32 ; 57 ; 60]$} \\
\hline 26 & Flavonol & Quercetin & $\mathrm{C}_{15} \mathrm{H}_{10} \mathrm{O}_{7}$ & 302.2357 & & 303 & $\begin{array}{l}285 ; 267 \\
163 ; 159\end{array}$ & {$[9 ; 12 ; 48 ; 50]$} \\
\hline 27 & Flavonol & $\begin{array}{l}\text { Dihydroquercetin } \\
\text { (Taxifolin; Taxifoliol) }\end{array}$ & $\mathbf{C}_{15} \mathrm{H}_{12} \mathrm{O}_{7}$ & 304.2516 & & 305 & $\begin{array}{c}259 ; 241 \\
199 ; 149\end{array}$ & {$[12 ; 48 ; 52]$} \\
\hline
\end{tabular}




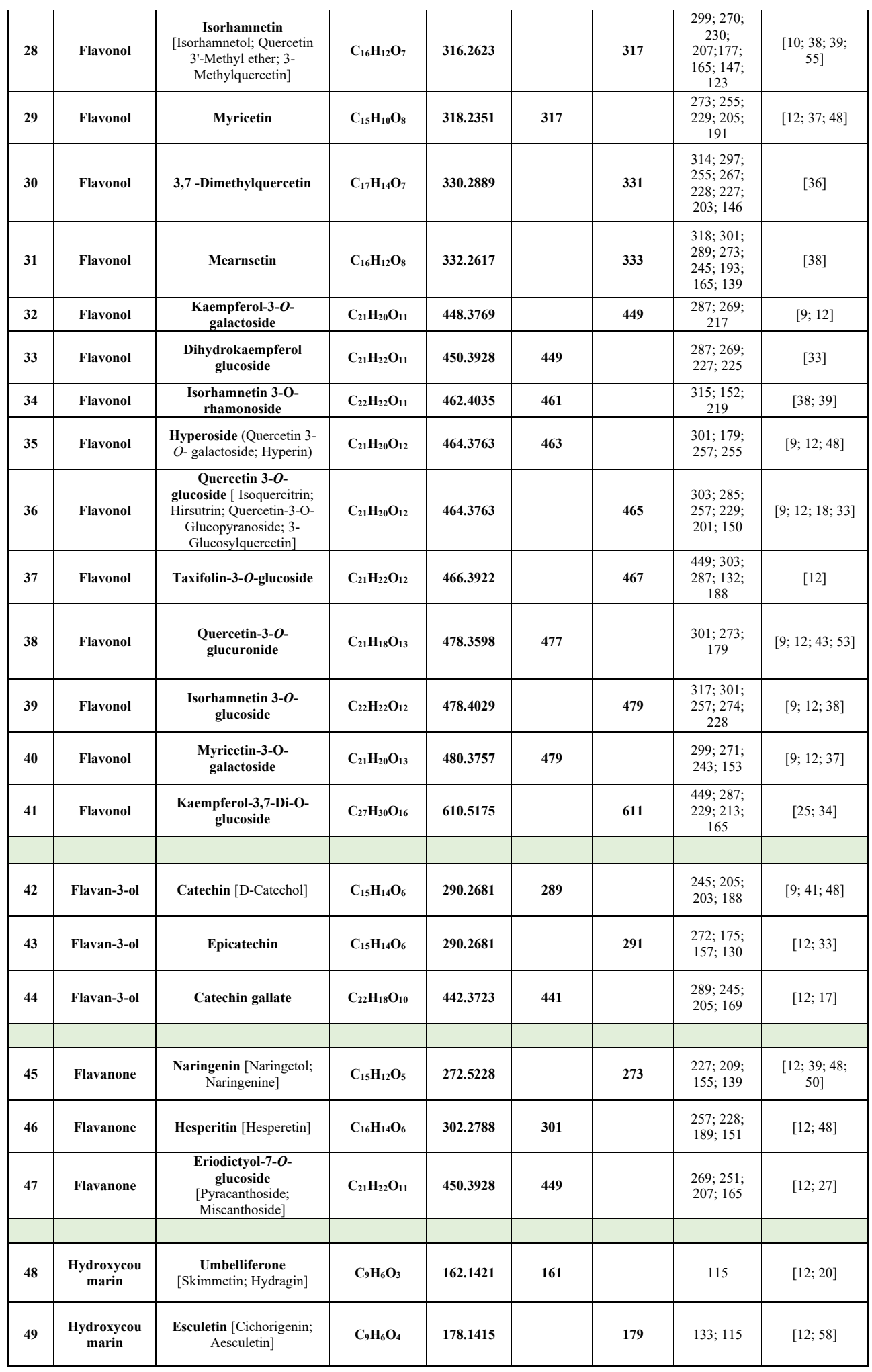




\begin{tabular}{|c|c|c|c|c|c|c|c|c|}
\hline 50 & $\begin{array}{l}\text { Hydroxycou } \\
\text { marin }\end{array}$ & $\begin{array}{c}\text { Esculin [Aesculin; } \\
\text { Esculoside; Polichrome] }\end{array}$ & $\mathrm{C}_{15} \mathrm{H}_{16} \mathrm{O}_{9}$ & 340.2821 & 339 & & $\begin{array}{c}177 ; 293 \\
131\end{array}$ & $\begin{array}{c}{[12 ; 13 ; 18 ;} \\
58]\end{array}$ \\
\hline 51 & Coumarin & $\begin{array}{c}\text { Fraxin (Fraxetin-8-O- } \\
\text { glucoside) }\end{array}$ & $\mathrm{C}_{16} \mathrm{H}_{18} \mathrm{O}_{10}$ & 370.3081 & & 371 & $\begin{array}{c}208 ; 352 ; \\
135 \\
\end{array}$ & {$[12]$} \\
\hline 52 & Coumarin & $\begin{array}{c}\text { Fraxetin-7-O-beta- } \\
\text { glucuronide }\end{array}$ & $\mathrm{C}_{16} \mathrm{H}_{16} \mathrm{O}_{11}$ & 384.2916 & & 385 & $\begin{array}{c}367 ; 272 \\
209 ; 175 ; \\
158 ; 143 \\
\end{array}$ & [59] \\
\hline 53 & Stilbene & $\begin{array}{c}\text { Resveratrol [trans- } \\
\text { Resveratrol; 3,4',5- } \\
\text { Trihydroxystilbene; } \\
\text { Stilbentriol] } \\
\end{array}$ & $\mathrm{C}_{14} \mathrm{H}_{12} \mathrm{O}_{3}$ & 228.2433 & & 229 & $\begin{array}{c}142 ; 184 \\
114\end{array}$ & {$[13 ; 48]$} \\
\hline 54 & Stilbene & $\begin{array}{l}\text { Polydatin [Piceid; trans- } \\
\text { Piceid] }\end{array}$ & $\mathrm{C}_{20} \mathrm{H}_{22} \mathrm{O}_{8}$ & 390.3839 & 389 & & $\begin{array}{c}227 ; 343 ; \\
184 \\
\end{array}$ & {$[33 ; 48]$} \\
\hline 55 & $\begin{array}{c}\text { Hydroxycin } \\
\text { namic acid }\end{array}$ & p-Coumaric acid & $\mathrm{C}_{9} \mathrm{H}_{8} \mathrm{O}_{3}$ & 164.16 & & 165 & $146 ; 119$ & {$[12 ; 33]$} \\
\hline 56 & $\begin{array}{c}\text { Hydroxycin } \\
\text { namic acid }\end{array}$ & $\begin{array}{c}\text { Caffeic acid [(2E)-3-(3,4- } \\
\text { Dihydroxyphenyl)acrylic } \\
\text { acid] }\end{array}$ & $\mathrm{C}_{9} \mathrm{H}_{8} \mathrm{O}_{4}$ & 180.1574 & 179 & & 133 & {$[12 ; 41]$} \\
\hline 57 & $\begin{array}{l}\text { Hydroxycin } \\
\text { namic acid }\end{array}$ & $\begin{array}{l}\text { Sinapic acid [trans- } \\
\text { Sinapic acid] }\end{array}$ & $\mathrm{C}_{11} \mathrm{H}_{12} \mathrm{O}_{5}$ & 224.21 & & 225 & $\begin{array}{l}179 ; 153 \\
133 ; 115\end{array}$ & {$[4 ; 12 ; 42]$} \\
\hline 58 & $\begin{array}{l}\text { Hydroxycin } \\
\text { namic acid }\end{array}$ & Caffeoylmalic acid & $\mathrm{C}_{13} \mathrm{H}_{12} \mathrm{O}_{8}$ & 296.2296 & 295 & & $\begin{array}{c}133 ; 179 \\
148 ; 119 \\
115 \\
\end{array}$ & [43] \\
\hline 59 & $\begin{array}{l}\text { Hydroxycin } \\
\text { namic acid }\end{array}$ & $\begin{array}{l}\text { Coutaric acid [Trans-P- } \\
\text { Coumaroyltartaric acid] }\end{array}$ & $\mathrm{C}_{13} \mathrm{H}_{12} \mathrm{O}_{8}$ & 296.2296 & 295 & & $163 ; 119$ & {$[12]$} \\
\hline 60 & $\begin{array}{l}\text { Hydroxycin } \\
\text { namic acid }\end{array}$ & $\begin{array}{c}\text { Caftaric acid [Cis- } \\
\text { Caftaric acid; 2-Caffeoyl- } \\
\text { L-Tartaric acid; Caffeoyl } \\
\text { Tartaric acid\} }\end{array}$ & $\mathrm{C}_{13} \mathrm{H}_{12} \mathrm{O}_{9}$ & 312.23 & 311 & & $\begin{array}{c}149 ; 221 \\
131\end{array}$ & {$[6 ; 12 ; 41]$} \\
\hline 61 & $\begin{array}{c}\text { Hydroxycin } \\
\text { namic acid }\end{array}$ & Fertaric acid [Fertarate] & $\mathrm{C}_{14} \mathrm{H}_{14} \mathrm{O}_{9}$ & 326.2556 & 325 & & $\begin{array}{c}193 ; 149 ; \\
134\end{array}$ & {$[12]$} \\
\hline 62 & $\begin{array}{l}\text { Hydroxycin } \\
\text { namic acid }\end{array}$ & $\begin{array}{c}p \text {-Coumaric acid-O- } \\
\text { hexoside [Trans- } p \text { - } \\
\text { Coumaric acid 4- } \\
\text { glucoside] }\end{array}$ & $\mathrm{C}_{15} \mathrm{H}_{18} \mathrm{O}_{8}$ & 326.2986 & 325 & & $\begin{array}{c}193 ; 163 \\
119\end{array}$ & {$[13 ; 37 ; 43]$} \\
\hline 63 & $\begin{array}{l}\text { Hydroxycin } \\
\text { namic acid }\end{array}$ & $\begin{array}{c}\text { 1-Caffeoyl-beta-D- } \\
\text { glucose [Caffeic acid- } \\
\text { glucoside] }\end{array}$ & $\mathrm{C}_{15} \mathrm{H}_{18} \mathrm{O}_{9}$ & 342.298 & 341 & & $\begin{array}{l}179 ; 161 \\
135\end{array}$ & {$[12 ; 31]$} \\
\hline 64 & $\begin{array}{c}\text { Hydroxycin } \\
\text { namic acid }\end{array}$ & $\begin{array}{l}\text { 5- } O \text {-(4'- } O \text {-p-coumaroyl } \\
\text { glucosyl)quinic acid }\end{array}$ & $\mathrm{C}_{22} \mathrm{H}_{28} \mathrm{O}_{13}$ & 500.4499 & & 501 & $\begin{array}{l}339 ; 277 \\
203\end{array}$ & [18] \\
\hline 65 & $\begin{array}{l}\text { Hydroxycin } \\
\text { namic acid }\end{array}$ & $\begin{array}{c}p- \\
\begin{array}{c}\text { Coumaroylcaffaoylquini } \\
\text { c acid }\end{array} \\
\end{array}$ & $\mathrm{C}_{25} \mathrm{H}_{24} \mathrm{O}_{11}$ & 500.4515 & & 501 & $\begin{array}{c}355 ; 483 \\
281 ; 225 \\
193 ; 181 \\
\end{array}$ & [49] \\
\hline 66 & $\begin{array}{c}\text { Hydroxycin } \\
\text { namic acid }\end{array}$ & $\begin{array}{c}\text { Coumaric acid } \\
\text { derivative }\end{array}$ & $\mathbf{C}_{30} \mathbf{H}_{30} \mathbf{O}_{7}$ & 502.5550 & & 503 & $\begin{array}{c}457 ; 411 ; \\
391 ; 367 ; \\
382 ; 339 ; \\
293 \\
\end{array}$ & [43] \\
\hline 67 & $\begin{array}{c}\text { Hydroxyben } \\
\text { zoic acid }\end{array}$ & Gallic acid & $\mathrm{C}_{7} \mathrm{H}_{6} \mathrm{O}_{5}$ & 170.1195 & & 171 & 126 & {$[12 ; 39]$} \\
\hline 68 & $\begin{array}{c}\text { Hydroxyben } \\
\text { zoic acid }\end{array}$ & $\begin{array}{l}\text { Ellagic acid [Benzoaric } \\
\text { acid; Elagostasine; } \\
\text { Lagistase; Eleagic acid] }\end{array}$ & $\mathrm{C}_{14} \mathrm{H}_{6} \mathrm{O}_{8}$ & 302.1926 & & 303 & $\begin{array}{c}172 ; 158 \\
144 ; 127 \\
116\end{array}$ & {$[12 ; 33 ; 47]$} \\
\hline 69 & $\begin{array}{l}\text { Hydroxyben } \\
\text { zoic acid }\end{array}$ & Salvianolic acid $F$ & $\mathrm{C}_{17} \mathrm{H}_{14} \mathrm{O}_{6}$ & 314.2895 & & 315 & $\begin{array}{l}269 ; 243 \\
213 ; 207 \\
185 ; 181 \\
153 ; 144\end{array}$ & [6] \\
\hline 70 & $\begin{array}{c}3,4- \\
\text { dihydroxybe } \\
\text { nzoic acid }\end{array}$ & $\begin{array}{l}\text { Dihydroxybenzoyl- } \\
\text { hexoside }\end{array}$ & $\mathrm{C}_{13} \mathrm{H}_{16} \mathrm{O}_{9}$ & 316.2607 & 315 & & $\begin{array}{c}153 ; 253 \\
284\end{array}$ & [31] \\
\hline 71 & $\begin{array}{l}\text { Hydroxyben } \\
\text { zoic acid }\end{array}$ & Salvianolic acid G & $\mathrm{C}_{18} \mathrm{H}_{12} \mathrm{O}_{7}$ & 340.2837 & & 341 & $\begin{array}{c}323 ; 295 \\
255 ; 195 \\
159 \\
\end{array}$ & {$[19 ; 56]$} \\
\hline 72 & $\begin{array}{l}\text { Hydroxyben } \\
\text { zoic acid }\end{array}$ & Salvianolic acid D & $\mathrm{C}_{20} \mathrm{H}_{18} \mathrm{O}_{10}$ & 418.3509 & 417 & & $\begin{array}{l}373 ; 329 \\
287 ; 209\end{array}$ & {$[5 ; 6]$} \\
\hline 73 & $\begin{array}{c}\text { Hydroxyben } \\
\text { zoic acid }\end{array}$ & $\begin{array}{c}\text { Dimethyl ellagic acid } \\
\text { hexose }\end{array}$ & $\mathrm{C}_{22} \mathrm{H}_{20} \mathrm{O}_{13}$ & 492.3864 & & 493 & $\begin{array}{c}331 ; 299 \\
270 ; 242 \\
179 ; 150 \\
\end{array}$ & [14] \\
\hline & & Others & & & & & & \\
\hline
\end{tabular}




\begin{tabular}{|c|c|c|c|c|c|c|c|c|}
\hline 74 & $\begin{array}{c}2- \\
\text { hydroxycar } \\
\text { boxylic acid }\end{array}$ & $\begin{array}{l}\text { Malic acid [DL-Malic } \\
\text { acid] }\end{array}$ & $\mathrm{C}_{4} \mathrm{H}_{6} \mathrm{O}_{5}$ & 134.0874 & 133 & & 115 & {$[6 ; 16 ; 43]$} \\
\hline 75 & $\begin{array}{l}\text { Dicarboxylic } \\
\text { acid }\end{array}$ & Tartaric acid & $\mathrm{C}_{4} \mathrm{H}_{6} \mathrm{O}_{6}$ & 150.09 & 149 & & 131 & [16] \\
\hline 76 & Auxin & Indole-3-carboxylic acid & $\mathrm{C}_{10} \mathrm{H}_{9} \mathrm{NO}_{2}$ & 175.1840 & & 176 & 130 & [36] \\
\hline 77 & $\begin{array}{l}\text { Tricarboxyli } \\
\text { c acid }\end{array}$ & $\begin{array}{c}\text { Citric acid [Anhydrous; } \\
\text { Citrate] }\end{array}$ & $\mathrm{C}_{6} \mathrm{H}_{8} \mathrm{O}_{7}$ & 192.1235 & 191 & & $\begin{array}{l}111 ; 173 \\
143 ; 127\end{array}$ & {$[28 ; 43 ; 47]$} \\
\hline 78 & $\begin{array}{c}\text { Polyhydroxy } \\
\text { carboxylic } \\
\text { acid }\end{array}$ & Quinic acid & $\mathrm{C}_{7} \mathrm{H}_{12} \mathrm{O}_{6}$ & 192.1666 & 191 & & $111 ; 173$ & {$[12 ; 13 ; 28]$} \\
\hline 79 & $\begin{array}{l}\text { Propionic } \\
\text { acid }\end{array}$ & Dihydroferulic acid & $\mathrm{C}_{10} \mathrm{H}_{12} \mathrm{O}_{4}$ & 196.1999 & 195 & & $\begin{array}{l}159 ; 129 \\
122 ; 113\end{array}$ & [13] \\
\hline 80 & Gallate ester & Ethyl gallate & $\mathrm{C}_{9} \mathrm{H}_{10} \mathrm{O}_{5}$ & 198.1727 & 197 & & $169 ; 125$ & [14] \\
\hline 81 & Amino acid & $\begin{array}{c}\text { L-Tryptophan } \\
\text { [Tryptophan; (S)- } \\
\text { Tryptophan] }\end{array}$ & $\mathrm{C}_{11} \mathrm{H}_{12} \mathrm{~N}_{2} \mathrm{O}_{2}$ & 204.2252 & & 205 & $\begin{array}{l}188 ; 146 \\
170 ; 118\end{array}$ & {$[31 ; 47]$} \\
\hline 82 & $\begin{array}{l}\text { Carboxylic } \\
\text { acid }\end{array}$ & Myristeolic acid & $\mathrm{C}_{14} \mathrm{H}_{26} \mathrm{O}_{2}$ & 226.3550 & & 227 & $\begin{array}{c}209 ; 199 \\
181 ; 155 \\
127\end{array}$ & [13] \\
\hline 83 & $\underset{\text { acid }}{\text { Omega-3 }}$ & $\begin{array}{l}\text { Linolenic acid (Alpha- } \\
\text { Linolenic acid; } \\
\text { Linolenate) }\end{array}$ & $\mathrm{C}_{18} \mathrm{H}_{30} \mathrm{O}_{2}$ & 278.4296 & & 279 & $\begin{array}{l}260 ; 176 \\
120\end{array}$ & [4] \\
\hline 84 & $\begin{array}{l}\text { Saturated } \\
\text { long-chain } \\
\text { fatty acid }\end{array}$ & $\begin{array}{c}\text { 9-Oxo-10E,12Z- } \\
\text { octadecanoic acid [9- } \\
\text { Oxo-ODE] }\end{array}$ & $\mathrm{C}_{18} \mathrm{H}_{30} \mathrm{O}_{3}$ & 294.4290 & & 295 & $\begin{array}{l}249 ; 220 \\
165 ; 125\end{array}$ & [13] \\
\hline 85 & $\begin{array}{l}\text { Phenolic } \\
\text { glycoside }\end{array}$ & $\begin{array}{c}\text { Protocatechuic acid-O- } \\
\text { hexoside }\end{array}$ & $\mathrm{C}_{13} \mathrm{H}_{16} \mathrm{O} 9$ & 316.2607 & 315 & & $\begin{array}{c}153 ; 151 ; \\
298\end{array}$ & {$[43 ; 50]$} \\
\hline 86 & Gallate ester & $\begin{array}{l}\text { Galloyl glucose [Beta- } \\
\text { Glucogallin; 1-O-Galloyl- } \\
\text { Beta-D-Glucose] }\end{array}$ & $\mathrm{C}_{13} \mathrm{H}_{16} \mathbf{O}_{10}$ & 332.2601 & 331 & & $\begin{array}{c}313 ; 195 ; \\
166\end{array}$ & [47] \\
\hline 87 & $\begin{array}{l}\text { Gallic acid } \\
\text { hexoside }\end{array}$ & Gallic acid hexoside & $\mathrm{C}_{13} \mathrm{H}_{16} \mathrm{O}_{10}$ & 332.2601 & 331 & & $\begin{array}{l}271 ; 169 \\
\quad 125\end{array}$ & [37] \\
\hline 88 & $\begin{array}{l}\text { Berberine } \\
\text { alkaloid }\end{array}$ & $\begin{array}{c}\text { Palmatine } \\
\text { [Berbericinine; } \\
\text { Burasaine] }\end{array}$ & $\mathrm{C}_{21} \mathrm{H}_{22} \mathrm{NO}_{4}$ & 352.4037 & & 353 & $\begin{array}{l}335 ; 317 \\
235 ; 137\end{array}$ & [58] \\
\hline 89 & $\begin{array}{l}\text { Amino } \\
\text { sugar }\end{array}$ & Hexose-hexose-N-acetyl & $\mathrm{C}_{14} \mathrm{H}_{25} \mathrm{NO}_{10}$ & 367.3490 & 366 & & $186 ; 142$ & {$[25]$} \\
\hline 90 & Sterol & $\begin{array}{l}\text { Fucosterol [Fucostein; } \\
\text { Trans-24- } \\
\text { Ethylidenecholesterol] }\end{array}$ & $\mathrm{C}_{29} \mathrm{H}_{48} \mathrm{O}$ & 412.6908 & & 413 & $\begin{array}{l}395 ; 355 ; \\
297 ; 271 ; \\
199 ; 268 \\
194 ; 119\end{array}$ & [13] \\
\hline 91 & Sterol & $\begin{array}{c}\text { Stigmasterol } \\
\text { [Stigmasterin; Beta- } \\
\text { Stigmasterol] } \\
\end{array}$ & $\mathrm{C}_{29} \mathrm{H}_{48} \mathrm{O}$ & 412.6908 & & 413 & $\begin{array}{l}301 ; 259 \\
189 ; 171\end{array}$ & [13] \\
\hline 92 & $\begin{array}{l}\text { Dihydrochal } \\
\text { cone }\end{array}$ & $\begin{array}{l}\text { Phlorizin [Phloridzin; } \\
\text { Phlorizoside; Floridzin: } \\
\text { phlorrhizin; Phloretin 2'- } \\
\text { Glucoside; Phloretin-O- } \\
\text { hexoside] }\end{array}$ & $\mathrm{C}_{21} \mathbf{H}_{24} \mathrm{O}_{10}$ & 436.4093 & & 437 & $\begin{array}{l}397 ; 377 \\
217\end{array}$ & {$[12 ; 13]$} \\
\hline 93 & Triterpene & Oleanoic acid & $\mathrm{C}_{30} \mathrm{H}_{48} \mathrm{O}_{3}$ & 456.7003 & & 457 & $\begin{array}{c}439 ; 411 \\
365 ; 364 \\
337 ; 309 \\
293 ; 248 \\
205 \\
\end{array}$ & {$[32 ; 49]$} \\
\hline 94 & Triterpene & Ursolic acid & $\mathrm{C}_{30} \mathrm{H}_{48} \mathrm{O}_{3}$ & 456.7003 & & 457 & $\begin{array}{l}411 ; 393 ; \\
365 ; 337 ; \\
279 ; 247 ; \\
219 ; 205\end{array}$ & {$[32 ; 49 ; 56]$} \\
\hline
\end{tabular}

Research carried out using tandem mass spectrometry showed the presence of 94 biologically active compounds corresponding to the species $V$. amurensis, moreover, salvianolic acids F, D and G, oleanolic, ursolic, myristoleic acids, berbericinin, mearnsetin, 
esculin, nevadensin, stigmasterol, fucosterol, phlorizin, L-tryptophan were identified for the first time in $\mathrm{V}$. amurensis.

Anthocyanins have been identified in extracts: Malvidin-3-O-glucoside, Pelargonidin-3$O$-glucoside (callistephin), Peonidin-3-O-glucoside, Cyanidin-3,5-dihexoside, Cyanidin-3,5diglucoside, Peonidin-3,5-diglucoside, Malvidin 3-(6- $O$-coumaroyl) glucoside, Petunidin-3$O$-glucoside-5-O-glucoside, Malvidin 3-(6'- $p$-caffeoyl glucoside), Malvidin 3,5-Odiglucoside. A large group of flavonoids identified; flavonols: Kaempferol, Aromadendrin, Kaempferide, Quercetin, Dihydroquercetin, Kaempferol-3-O-galactoside, Quercetin 3-Ogalactoside, Taxifolin-3-O-glucoside, Quercetin-3-O-glucuronide, Isorhamnetin-3-Orhamonoside, Isorhamnetin-3-O-glucoside, Myricetin-3-O-galactoside, Kaempferol-3,7-Di$O$-glucoside; flavones: Apigenin, Luteolin, Nevadensin, Apigenin-7-O-glucoside, Isovitexin 6"-O-deoxyhexoside, Vitexin 2"-O-glucoside, Apigenin 6-C-[6"-acetyl-2"-Odeoxyhexoside]-glucoside; flavanones: Naringenin, Hesperitin, Eriodictyol-7-O-glucoside; flavan-3-ols: Catechin, Epicatechin.

Glycosylated coumarins have also been identified: Umbelliferone, Esculin, Fraxin, Fraxetin-7-O-beta-glucuronide, berberine Palmatine, stilbenes Polydatin and transResveratrol, sterols: Fucosterol, Stigmasterol, dihydrochalcone Phlorizin.

Отдельно необходимо отметить, что такие соединения, как кумарины Umbelliferone, Fraxin и Esculin, флавон Nevadensin, флаван-3-ол Epicatechin, стерол Fucosterol, флаванол Taxifolin-3-O-glucoside были идентифицированы с помощью масс-спектрометрического исследования только в островных образцах дикого винограда $V$. amurensis (острова Пахтусова и остров Рикорда, залив Петра Великого, Японское море).

Separately, it should be noted that compounds such as coumarins Umbelliferone, Fraxin and Esculin, flavone Nevadensin, flavan-3-ol Epicatechin, sterol Fucosterol, flavanol Taxifolin-3-O-glucoside were identified by mass spectrometric studies only in island samples of wild $V$. amurensis grapes (Pakhtusov Islands and Rikord Island, Peter the Great Bay, Japanese Sea).

\section{Conclusions}

Amur grape $V$. amurensis Rupr. contains a large number of polyphenolic complexes, which are biologically active compounds. In this work, we have tried for the first time to carry out a comparative metabolic study of biologically active substances of wild grapes obtained from five different places in the Primorsky and Khabarovsk territories. High performance liquid chromatography (HPLC) in combination with a BRUKER DALTONIKS ion trap (tandem mass spectrometry) was used to identify target analytes in extracts. The results showed the presence of 94 biologically active compounds corresponding to the species $\mathrm{V}$. amurensis, and salvianolic acids F, D and G, oleanolic, ursolic, myristeolic acids, berbericinin, mearnsetin, esculin, nevadensin, stigmasterol, fucosterol, phlorizin, L-tryptophan were identified for the first time in $V$. amurensis.

The findings may support future research into the production of various pharmaceutical and dietary supplements containing $V$. amurensis extracts. A wide variety of biologically active polyphenolic compounds opens up rich opportunities for the creation of new drugs and biologically active additives based on extracts from this family of grapes (Vitaceae).

\section{References}

1. Ampelography USSR 494 (Moscow, Pishchepromizdat, 1946) (In Russian)

2. E.W. Berry, Lower Cretaceous (Baltimore, Maryland Geological Survey, 1911) 
3. E.W. Berry, Upper Cretaceous floras of the World. Text and atlas (Baltimore, Maryland Geological Survey, 1917)

4. W. Chen, L. Gong, Z. Guo, W. Wang, H. Zhang, X. Liu, S. Yu, L. Xiong, J. Luo, Molecular Plant 6(6), 1769-1780 (2013)

5. X. Chen, S. Zhang, Z. Xuan, D. Ge, X. Chen, J. Zhang, Q. Wang, Y. Wu, B. Liu, Molecules, 22, 811 (2017)

6. M. Cirlini, P. Mena, M. Tassotti, K.A. Herrlinger, K.M. Nieman, C. Dall'Asta, D. Del Rio, Molecules, 21, 1007 (2016)

7. L.P. da Silva, E. Pereira, T.C.S.P. Pires, M.J. Alves, O.R. Pereira, L. Barros, I.C.F.R. Ferreira, Food Res. Int., 119, 34-43 (2019)

8. A. Daikonya, S. Kitanaka, Japan. J. Food Chem. Safety 18(3), 183-190 (2011)

9. M. De Rosso, L. Tonidandel, R. Larcher, G. Nicolini, A. Dalla Vedova, F. De Marchi, M. Gardiman, M. Giust, R. Flamini, Food Chem. 163, 244-251 (2014)

10. C. Fu, P. Yu, M. Wang, F. Qiu, Food Chem., 312, 126070 (2020)

11. M. Garg, M. Chawla, V. Chunduri, R. Kumar, S. Sharma, N.K. Sharma, N. Kaur, A. Kumar, J.K. Mundey, M.K. Saini, S.P. Singh, J. Cereal Sci. 71, 138e144 (2016)

12. P. Goufo, R.K. Singh, I. Cortez, Antioxidants 9, 398 (2020)

13. A.R. Hamed, S.S. El-Hawary, R.M. Ibrahim, U.R. Abdelmohsen, A.M. El-Halawany, J. Chrom. Sci. 0, 1-9 (2020)

14. F. Han, Y. Li, L. Ma, T. Liu, Y. Wu, R. Hu, A. Song, R. Yin, Talanta, 160, 183-193 (2016)

15. Hegi G. Vitaceae (Ampelidaceae) Rebengewachse, Illustrierte Flora von Mittel-Europa Munchen, 5, 1, 350-426 (1925)

16. V. Ivanova-Petropulos, Z. Naceva, V. Sandor, L. Makszin, L. Deutsch-Nagy, B. Berkics, T. Stafilov, F. Kilar, Electrophoresis 39, 1597-1605 (2018)

17. R. Jaiswal, L. Jayasinghe, N. Kuhnert, J. Mass Spectrom. 47, 502-515 (2012)

18. R. Jaiswal, H. Muller, A. Muller, M.G.E. Karar, N. Kuhnert, Phytochem. 108, 252-263 (2014)

19. R.-W. Jiang, K.-M. Lau, P.-M. Hon, T.C.W. Mak, K.-S. Woo, K.-P. Fung, Current Med. Chem. 12, 237-246 (2005)

20. S. Kim, S. Oh, H.B. Noh, S. Ji, S.H. Lee, J.M. Koo, C.W. Choi, H.P. Jhun, Molecules, 23, 3001 (2018)

21. A. N. Krishtofovich, Proceedings of the Geological Prospecting Institute 98, 5 (1931) (In Russ.)

22. A. N. Krishtofovich, Botanical journal of the USSR, 23 (5-6), 365-375 (1938) (In Russ.)

23. A. N. Krishtofovich, M. I. Borsuk, Paleontological problems, 5, 375-396 (1939) (In Russ.)

24. E.S. Lago-Vanzela, R. Da-Silva, E. Gomes, E. Garcia-Romero, E. Hermosin-Gutierres, Agric. Food Chem. 59, 13136-13146 (2011)

25. G. Le Gall, M.S. DuPont, F.A. Mellon, A.L. Davis, G.J. Collins, M.E. Verhoeven, I.J. Colquhoun, J. Agricult. Food Chem. 51, 2438-2446 (2003)

26. T.H. Lee, C.C. Hsu, G. Hsiao, J.Y. Fang, W.M. Liu, C.K. Lee, Planta Med. 82(8), 698704 (2016)

27. X. Li, T. Tian, Frontiers in Pharm, 9, 1067 (2018) 
28. M.M. Marzouk, S.R. Hussein, A. Elkhateeb, M. El-shabrawy, E.-S. S. Abdel-Hameed, S.A. Kawashty, J. Applied Pharm. Sci. 8(08), 116-122 (2018)

29. I.V. Michurin, New village, I-II, (1929-1932) (In Russian)

30. I.V. Michurin, Bulletin of gardening, fruit growing and horticulture, 4-5 (1907) (In Russian)

31. M. Ozarowski, A. Piasecka, A. Paszel-Jaworska, Siqueira de A. Chaves, D., A. Romaniuk, M. Rybczynska, A. Gryszczynska, A. Sawikowska, P. Kachlicki, P.L. Mikolajczak, A. Seremak-Mrozikiewicz, Braz. J. Pharmacol. 28, 179-191 (2018)

32. R. Pandey, B. Kumar, J. Liquid Chromatogr. \& Related Technol. 39, 225-238 (2016)

33. L. Paudel, F.J. Wyzgovski, J.C. Scheerens, A.M. Chanon, R.N. Reese, D. Smiljanic, C. Wesdemiotis, J.J. Blakeslee, K.M. Riedl, P.L. Rinaldi, J. Agricult. Food. Chem. 61, 12032-12043 (2013)

34. A. Petsalo, J. Jalonen, A. Tolonen, J Chromatogr. A. 1112(1-2), 224-231 (2006)

35. P.C. Pradhan, S. Saha, J Food Sci. Technol. 53(2), 1205-1213 (2016)

36. P. Quifer-Rada, A. Vallverdu-Queralt, M. Martinez-Huelamo, G. Chiva-Blanch, O. Jauregui, R. Estruch, R. Lamuela-Raventos, Food Chem. 169, 336-343 (2015)

37. N. Rafsanjany, J. Senker, S. Brandt, U. Dobrindt, A. Hensel, J. Agric. Food Chem. 63, 8804-8818 (2015)

38. S.A.O. Santos, C.S.R. Freire, M.R.M. Domingues, A.J.D. Silvestre, C.P. Neto, J. Agric. Food Chem. 59, 9386-9393 (2011)

39. S.A.O. Santos, C. Vilela, C.S.R. Freire, C.P. Neto, A.J.D. Silvestre, J. Chromatogr. B. 938, 65-74 (2013)

40. A.J. Seukep, Y.-L. Zhang, Y.-B. Hu, M.-Q. Guo, Pharmaceut, 13, 59 (2020)

41. K. Schoedl, A. Forneck, M. Sulyok, R. Schuhmacher, J. Agric. Food. Chem. 59, $10787-$ 10794 (2011)

42. M. Sharma, R. Sandhir, A. Singh, P. Kumar, A. Mishra, S. Jachak, S.P. Singh, J. Singh, J. Roy, Front. Plant. Sci., 7, 1870 (2016)

43. V. Spinola, J. Pinto, P.C. Castilho, Food Chemistry 173, 14-30 (2015)

44. V.G. Sprygin, N.F. Kushnerova, Iu. A. Rakhmanin, Gig. Sanit. 3, 57-60 (2003) [in Russ.]

45. State Pharmacopeia XIV (2018) [in Russ.]

46. M. Suarez, A. Macia, M.-P. Romero, M.-J. Motiva, J. Chromatogr. A. 1214, 90-99 (2008)

47. J. Sun, X. Liu, T. Yang, J. Slovin, P. Chen, Food Chem., 146, 289-298 (2014)

48. J. Sun, F. Liang, Y. Bin, P. Li, C. Duan, Molecules 12, 679-693 (2007)

49. L. Sun, S. Tao, S. Zhang, Molecules 24, 159 (2019)

50. A. Vallverdu-Queralt, O. Jauregui, A. Medina-Remon, R.M. Lamuela-Raventos, Agricult. Food Chem. 60, 3373-3380 (2012)

51. N.I. Vavilov, Origin and geography of cultivated plants, 440 (Science, Petersburg, 1987) (In Russ.)

52. I.V. Voskoboinikova, N.A. Tjukavkina, S.V. Geodakyan, Y.A. Kolesnik, V.K. Kolhir, V.A. Zjuzin, S.J. Sokolov, Pharmacokinetics of dihydroquercetin. Phytotherapy Res. 7, 208-210 (1993)

53. H. Wang, E.J. Race, A.J. Shrikhande, Agric. Food Chem. 51, 1839-1844 (2003) 
54. A. Wojakowska, J. Perkowski, T. Goral, M. Stobiecki, J. Mass. Spectrom. 48, 329-339 (2013)

55. J. Xiao, T. Wang, P. Li, R. Liu, Q. Li, K. Bi, J. Chromatogr. B.1028, 33-41 (2016)

56. L. L. Xu, J. J. Xu, K. R. Zhong, Z. P. Shang, F. Wang, R. F. Wang, B. Liu, Molecules, 22, 1756 (2017)

57. X. Xu, B. Yang, D. Wang, Y. Zhu, X. Miao, W. Yang, Molecules 25, 4612 (2020)

58. L. Yang, X. Meng, X. Yu, H. Kuang, J. Pharm. Biomed. Analys. 134, 43-52 (2017)

59. T. Yasuda, M. Fukui, T. Nakazawa, A. Hoshikawa, K. Ohsawa, J. Nat. Prod. 69, 755757 (2006)

60. W.-H. Zhang, I.-Ch. Chao, D.-J. Hu, F. Shakerian, J. AOAC Int., 102(3) (2019) 